\title{
THE MAGNETIC FIELD STRENGTH IN THE LARGE MAGELLANIC CLOUD
}

\author{
M.E. COSTA ${ }^{\dagger}$, P.M. McCULLOCH and P.A. HAMILTON \\ Physics Dept, University of Tasmania, GPO Box 252C, Hobart, Australia 7000 \\ ${ }^{\dagger}$ Present address: Physics Dept, University of Western Australia, Nedlands, Australia 6009
}

\begin{abstract}
We have measured a value of $4 \pm 5 \mathrm{~m}^{-2}$ rad for the rotation measure of the radio pulsar PSR0529-66 in the LMC and, after allowing for the dispersion and rotation measures of our Galaxy on the pulsar's line of sight, we deduce that the magnetic field strength in the LMC is in the range 0 to $5 \mu$ Gauss oriented away from the Sun.
\end{abstract}

\section{Introduction}

The radio pulsar PSR0529-66 in the Large Magellanic Cloud (hereafter LMC) was discovered by McCulloch et al.(1983). For the present work polarization data was collected from this pulsar at the Parkes 64metre radiotelescope during 1988 March and with that data we have determined the rotation and dispersion measures of the pulsar to be $4 \pm 5 \mathrm{~m}^{-2} \mathrm{rad}$ and $100 \mathrm{~cm}^{-3} \mathrm{pc}$, respectively.

The mean component of the magnetic field parallel to the line of sight to the pulsar can be computed using the standard relation,

$$
\mathrm{B}_{/ /}=1.232 \mathrm{RM} / \mathrm{DM} \mu \mathrm{Gauss} \text {, }
$$

where RM and DM are the rotation and dispersion measures in $\mathrm{m}^{-2} \mathrm{rad}$ and $\mathrm{cm}^{-3} \mathrm{pc}$, respectively (Manchester and Taylor 1977). For the LMC pulsar the average magnetic field strength will include contributions from our Galaxy and intergalactic space as well as the LMC. So before we can determine the magnetic field strength in the LMC we have first to estimate the rotation and dispersion measures in the LMC on the pulsar's line of sight.

\section{Rotation and Dispersion Measures of PSR0529-66 in the LMC}

The Galactic pulsars closest to the line of sight of PSR0529-66 are PSR0403-76, PSR0538-75, and PSR0743-53 with rotation measures $16 \pm 10 \mathrm{~m}^{-2} \mathrm{rad}, 24 \pm 2 \mathrm{~m}^{-2} \mathrm{rad}$ and $-75 \pm 5 \mathrm{~m}^{-2} \mathrm{rad}$, respectively. We will ignore the rotation measure of PSR0743-53 because its line of sight passes through the Gum nebula and so its rotation measure is affected by the magnetic field and high electron density of that nebula. The weighted mean rotation measure of the other two pulsars is about $23 \pm 2 \mathrm{~m}^{-2} \mathrm{rad}$.

Simard-Normandin et al.(1981) have published rotation measures of extragalactic sources, including eight within 20 degrees of PSR0529-66 which have a weighted, mean rotation measure

101

R. Haynes and D. Milne (eds.), The Magellanic Clouds, 101-102.

(C) 1991 IAU. Printed in the Netherlands. 
of $24 \pm 20 \mathrm{~m}^{-2} \mathrm{rad}$. For all but one of these the rotation measure is positive, indicating that there is little or no Faraday rotation intrinsic to the sources themselves. The agreement between the rotation measures of the extragalactic sources and the foreground Galactic pulsars also suggests that little Faraday rotation occurs in the Galactic halo or intergalactic space. Hence the Galactic contribution to the rotation measure of PSR0529-66 is about $20 \pm 20 \mathrm{~m}^{-2} \mathrm{rad}$ and therefore the rotation measure on the path in the LMC is about $-20 \pm 20 \mathrm{~m}^{-2} \mathrm{rad}$.

The Galactic dispersion measure on the line of sight can be estimated in two ways. First, by integrating the Galactic electron density (Reynolds 1989) in the direction of PSR0529-66 to the distance of the LMC gives a dispersion measure of $70 \mathrm{~cm}^{-3} \mathrm{pc}$. Second, McConnell (1990 private communication) has studied the dispersion measures of a number of high latitude pulsars and deduced that the Galactic dispersion measure obeys the relation $\mathrm{DM} \approx 35 / \sin |b| \mathrm{cm}^{-3} \mathrm{pc}$, for sufficiently large $\mathrm{l} b \mathrm{l}$. For PSR0529-66 this gives a value of $\sim 65 \mathrm{~cm}^{-3} \mathrm{pc}$. Allowing for anomalies in the electron distribution, the actual Galactic dispersion measure on this line of sight probably lies in the range 50 to $100 \mathrm{~cm}^{-3} \mathrm{pc}$.

Savage and de Boer (1979) estimate that the Galactic halo has an electron density of less than about $10^{-3} \mathrm{~cm}^{-3}$ - and a thickness of $\sim 3 \mathrm{kpc}$, leading to a dispersion measure of $\sim 3 \mathrm{~cm}^{-3} \mathrm{pc}-$ negligible in the context of this discussion. The absence of any known source of strong ionising radiation in the intergalactic region suggests very low concentrations, if any, of free electrons. So it is probably reasonable to assume that the dispersion measure of the intergalactic medium is also negligible.

Given the possible range of dispersion measures in our Galaxy, a reasonable lower limit for the dispersion measure in the LMC is $10 \mathrm{~cm}^{-3} \mathrm{pc}$, and $50 \mathrm{~cm}^{-3} \mathrm{pc}$ is a generous upper limit.

\section{The magnetic field strength in the LMC}

Using equation (1), the rotation measure estimate $-20 \pm 20 \mathrm{~m}^{-2} \mathrm{rad}$ and the dispersion measure limits 10 to $50 \mathrm{~cm}^{-3} \mathrm{pc}$, the line-of-sight component of the magnetic field strength in the LMC is in the range 0 to $5 \mu$ Gauss and is pointing away from the Sun. These figures compare with the range $\sim 3$ to $\sim 10 \mu$ Gauss for the large-scale magnetic field strengths in our own and other galaxies (Sofue et al. 1986).

The rotation measure of PSR0529-66 may be dominated by some magnetic irregularity in the LMC since it is in an active star forming region rich in population I stars which are well noted for having massive stars, supernova remnants and other magnetic field and free electron concentrations. Our estimate is therefore a tentative indication of the strength of the large-scale magnetic field in the LMC and is the first such estimate.

\section{References}

Manchester, R.N., Taylor, J.H. (1977), Pulsars, Freeman and Co.

McCulloch, P.M., Hamilton, P.A., Ables, J.G., Hunt, A.J. (1983), Nature . 303, 307.

Reynolds, R.J. (1989), Astrophys. J. 339, L29.

Savage, B.D., de Boer, K.S. (1979), Astrophys. J. 230, L77.

Simard-Normandin, M., Kronberg, P.P., Button, S. (1981), Astrophys. J. Suppl. Ser. 45, 97.

Sofue, Y., Fujimoto, M., Wielebinski, R. (1986), Ann. Rev. Astron. Astrophys. 24, 459. 